\title{
Determination of her2 overexpression in patients with osteosarcoma
}

\begin{abstract}
Osteosarcomas accounts for $56 \%$ of bone tumors and $15 \%$ of all extra cranial solid tumors, with as much as $50 \%$ occurring in patient under 20 years old. The present retrospective study aim to evaluate if osteosarcomas overexpress Human Epidermal Growth Factor Receptor 2 oncogene, analyze whether it can be an important prognostic biomarker in bone tumors and to determine whether its expression is associated with disease-free periods and overall survival. 48 patients were included in the study, the sample was processed for hematoxylin and eosin, Human Epidermal Growth Factor Receptor 2 using immunohistochemistry, and fluorescent in situ hybridization for Human Epidermal Growth Factor Receptor 2 amplification in cases with an indeterminate result (score $2+$ ) by immunohistochemistry. With a mean follow-up of 38.1 months, the study revealed that there was $0 \%$ of patient with expression of Her2 by either fluorescent in situ hybridization or immunohistochemistry, with only 1 patient expressing a score of Human Epidermal Growth Factor Receptor 2 of $1+$, and the remaining with a score of 0 . There was no correlation between Her2 expression, survival, response to treatment or development of metastatic disease.
\end{abstract}

Keywords: osteosarcoma, receptor, epidermal growth factor, bone neoplasms, monoclonal antibody
Volume 4 Issue 2 - 2018

\author{
Zaki Antonio Taissoun-Aslan,' Luis A \\ Herrera- Montalvo, ${ }^{2}$ José de la Luz Díaz- \\ Chávez, ${ }^{3}$ David Cantú- León, ${ }^{4}$ Antonio \\ Gomez-Pedraza,' Mónica Lizzette Serrano- \\ Arevalo, ${ }^{5}$ Andrea Moreno-Tobon, ${ }^{6}$ Mateo \\ Mejia - Saldarriaga ${ }^{7}$ \\ 'Department of Surgery, National Cancer Institute, Mexico \\ ${ }^{2}$ Research Director, National Cancer Institute, Mexico \\ ${ }^{3}$ Basic Research Department, National Cancer Institute, Mexico \\ ${ }^{4}$ Surgical Research Department, National Cancer Institute, \\ Mexico \\ ${ }^{5}$ Department of Pathology, National Cancer Institute, Mexico \\ ${ }^{6}$ Department of Surgery, University of Antioquia, Colombia \\ ${ }^{7}$ Jacobi Medical Center, Internal Medicine Department, USA
}

Correspondence: Zaki Antonio Taissoun Aslan, Department of Surgery, National Cancer Institute, Mexico, Av San Fernando, Sección XVI,Tlalpan, CP I4080, Mexico, Tel +57 3043 955202, Email info@doctorzaki.com

Received: August 26, 2017 | Published: April 05, 2018
Abbreviations: CEP 17, chromosome enumeration probe 17; DAB, diaminobenzidine; EGFR, epidermal growth factor receptor; FISH, fluorescent in situ hybridization; H\&E, hematoxylin and eosin; her2/neu, human epidermal growth factor receptor 2; HR, hormone receptors; IHC, immunohistochemistry; OS, osteosarcoma; PBS, phosphate-buffered saline; PCR, polymerase chain reaction; $P D-l 1$, tumor programed cell death ligand 1

\section{Introduction}

Osteosarcoma (OS) are the most frequent bone cancer, with an annual estimated incidence in the United States of around 1000 cases in $2017 .{ }^{1}$ Around $40-50 \%$ of these cases arise in patient younger than 20 years, being the fifth most common tumor in this age group. ${ }^{2}$ OS also present a bimodal incidence curve, with a second peak around 65 years. ${ }^{3,4}$ In Mexico, 189 cases of OS were reported between 20052010 in patients younger than 20 years, with a net mortality of 0.37 per 100.000 inhabitants. ${ }^{5}$ OS is slightly more common in males, with male: female ratio of $1.4 / 1$ and is more common in patient who are caucasic. ${ }^{3}$

Several biomarkers for both prognostication and treatment have been postulated in OS with inconclusive results in the last years. Similar to the experience with breast cancer, the expression of hormone receptors (HR) for progestogens and estrogen and it's possible impact in development of OS has been described in vitro and in tissue samples. ${ }^{6}$ However inconsistent results have been also reported, with different variables affecting the expression of HR, being almost absent in the cranio-facial type. ${ }^{7}$ Another possible biomarker identified is Her2/neu, which is part of the family of the transmembrane, epidermal growth factor receptors (which include EGFR, Her2/neu, Her3 and Her4), and is involved in the transmission and maintenance of cell adhesion, survival, migration and survival. Distinctively, Her2 do not have a ligand, and its dimerization occurs by random interaction with other monomers of the epidermal growth factor receptor family and other Her2 monomers, leading to hetero/ homodimerization and promotion of cellular survival. ${ }^{8}$

Currently, the degree of Her2 overexpression in OS is unclear, since there are conflicting results. Some report 42-57\% Her2 overexpression in OS, with a significant impact in patient outcomes, including overall survival and metastasis rate. ${ }^{9-14}$ However, other researcher reported no overexpression of Her2 in OS, and no impact in metastasis rate or survival. ${ }^{15,16}$

This study aims to provide further information in this controversial subject, using the determination of Her2 overexpression by means of Immunohistochemistry (IHC) and fluorescent in-situ hybridization in cases of undetermined IHC. Additionally, a correlation of Her2 overexpression and clinical outcomes, such as metastasis rate, recurrence free survival and overall survival in this population with a unique ethnic and race background (Hispanic-Mexican).

\section{Materials and methods}

Informed consent was obtained from all individual participants included in the study. Patients who were diagnosed with OS of the pelvis and/or extremities between January of 2000 and June 2010 were included. A total of 52 patients were identified, however four (4) of them were excluded. Three of them were excluded since they did not receive care in the study institution, and the remaining due to poor 
follow up. All patients were treated in a multidisciplinary group at a national reference institution (In stitu to Nacional de Cancerologia, Mexico).

Pretreatment tissue samples were obtained from all patients that were included in the study. All specimens were evaluated at the treating reference center with hematoxylin and eosin (H\&E) stain, and for Her2 overexpression initially with IHC, and later with fluorescent in situ hybridization (FISH) in case of an indeterminate results (cases with $2+$ score in IHC). Figure 1 for IHC score used. The IHC score was based on membrane staining of Her2, as it has been previously described.

Figure I Kaplan Meier Curve describing overall survival.

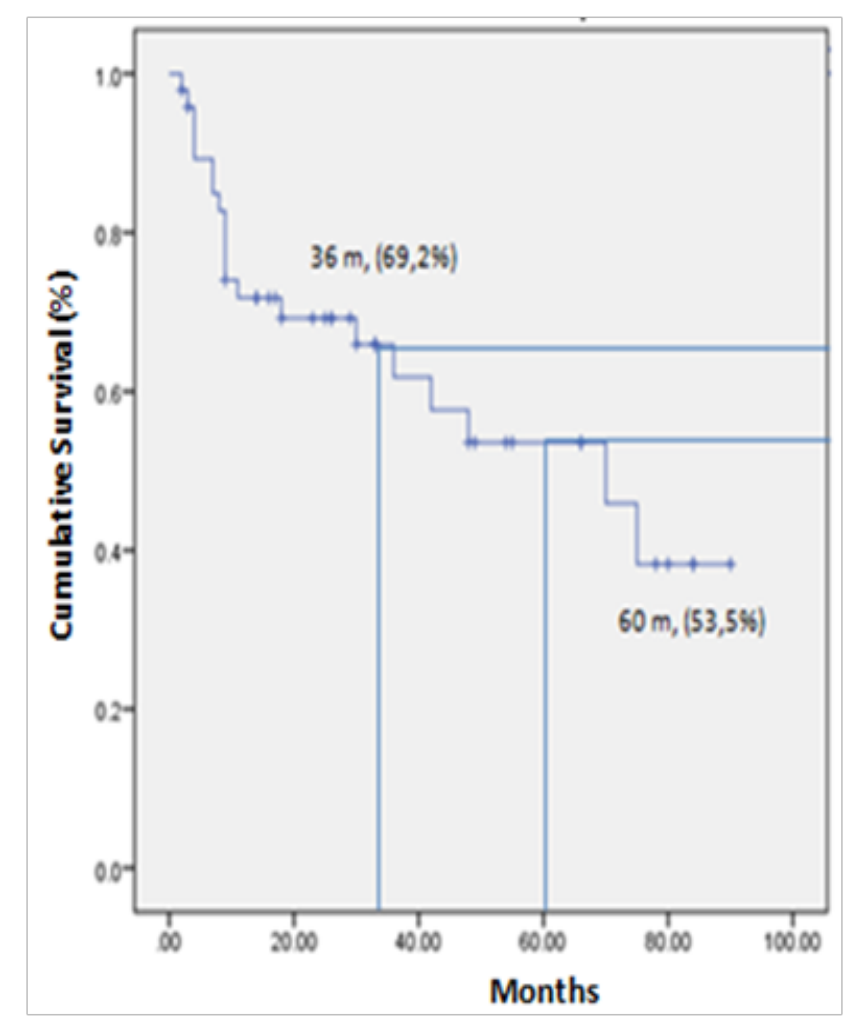

\section{Immunohistochemistry}

Slides were dewaxed using an oven for $30 \mathrm{~min}$ at $65^{\circ} \mathrm{C}$. Hydration with Xylene for 20 minutes was performed twice, and then $100 \%$ ethanol for $10 \mathrm{~min}$ was used. Finally, samples were washed with distilled water. Antigen retrieval was performed placing the slides in a Coplin plastic container with $25 \mathrm{ml}$ of TRIS-EDTApH9 buffer at a temperature of $92{ }^{\circ} \mathrm{C}$ for 40 minutes. Slides were cooled at room temperature and were incubated for 10min in PBS $1 \mathrm{X}$.

In order to eliminate the non-specific background, the samples were incubated in BSA 5\% solution in moisture chamber for 1 hour and washed with PBS-Triton $0.1 \%$ for 3 minutes. Then, slides were left overnight incubating in a moisture chamber with the primary antibody anti-Her2 (clone 29D8, Cell Signaling Technology) in a dilution of 1:500 buffered with PBS 1X. On the following morning, slides were washed 3 times for 3 minutes using $0.1 \%$ PBS-Triton and incubated again with PBS $1 \mathrm{X}$ for 5minutes.
To inactivate the endogenous peroxidase activity, slides were incubated in a solution of $3 \%$ hydrogen peroxide (Sigma-Aldrich, 323381 ) and absolute methanol using a 1:10 ratio for 30 minutes. Again, slides were washed 3 times for 3 minutes using $0.1 \%$ PBSTriton and incubated again with PBS $1 \mathrm{X}$ for 5 minutes. The slides were incubated with the biotinylated secondary antibody (Dako) for $30 \mathrm{~min}$. After three washes with $0.1 \%$ PBS-Triton buffer, the slides were incubated with streptavidin HRP complex for 30 minutes. After 3 washes with PBS-1X buffer, slides were exposed to diaminobenzidine (DAB, Dako) and finally contrasted with hematoxylin. All slides covered with Entellan (Merk, 107960) cover slips. All slides included previously identified positive and negative controls.

The statistical analysis was performed using descriptive statistics, using measures of central trend (mean and standard deviation) for quantitative and qualitative variables, respectively. Parametric and non-parametric statistics was used to compare variables after assessing the distribution of each variable.

For categorical variables, chi-squared test (or exact Fisher test when conditions were not met) was used. All the analysis was performed using SPSS statistical package, version 20 (IBM corporation). There were no violations of the ethics code as delineated by Helsinki declaration in its last review by the world medical association on October 2000. Since this was a retrospective and descriptive study, no patient was at risk or was subjected to any change in conduct. The institutional review board review the study protocol and approved the study.

\section{Results}

48 patients were included, all them had adequate tissue sample, 36 were men $(66.7 \%)$ and $16(33.3 \%)$ were women, with a mean age of 22.8 years (range 15-47 year). The histologic specimen was obtained by biopsy in $25(52.1 \%)$ cases, and after resection of the lesion in 23 $(47.9 \%)$. The most common affected site was the femur, in 30 patients $(62.5 \%)$, followed by tibia, $10(20.9 \%)$, forearm, 4 patients $(8.4 \%)$, humerus, 3 patients $(4.2 \%)$, and pelvis in 1 patient $(2.1 \%)$ (Table 1$)$.

Table 2 describes what type of surgical intervention the patient had, and use of neoadjuvant and adjuvant chemotherapy, and its complications. Patient who received neoadjuvant chemotherapy (25 patients) had 23 (47.9\% of total number of patient) partial response. Adjuvant chemotherapy was prescribed to 34 patients $(70.8 \%)$, mainly with regimens involving multiple medications.

The mean follows up time was 38.1 months (range: 3-90 months), distant relapse presented in 31 patients $(64.5 \%)$, being lung the most frequent site of relapse (23 patients). The mean recurrence free survival was 25.6 months (range: $0-90$ months), and the mean overall survival was 31.4 months (range: 2-90 months). 3 and 5-year survival was $69.2 \%$ and $53.5 \%$, respectively (Figure 2 ). Of the studied cohort, 20 patients $(41.7 \%)$ died during follow up.

After using IHC to determine Her2 overexpression in this cohort, $0 / 48$ patients $(0 \%)$ had expression of Her 2 . There was only 1 patient who had a score of $1+$, and the rest of the cohort had a IHC Her2 score of 0. Her 2-expression had no impact in relapse free survival or overall survival.

After the statistical analysis, only the clinical stage affected the relapse free period in this in this cohort $(\mathrm{p}<0.0)$, with higher clinical stage associated with lower relapse free period. 
Table I Patient characteristics and demographic information

\begin{tabular}{|c|c|}
\hline Sex & Patients (\%) \\
\hline Male & $32(66.7)$ \\
\hline Female & $16(33.3)$ \\
\hline $\begin{array}{l}\text { Signs and Symptoms Upon } \\
\text { Presentation }\end{array}$ & Patients (\%) \\
\hline Pain & $39(8 I .1)$ \\
\hline Palpable mass & $20(41.7)$ \\
\hline Impaired Function & $17(35.4)$ \\
\hline Deformity & $15(3 \mid .3)$ \\
\hline Pathologic Fracture & $9(18.8)$ \\
\hline Histologíc Subtype & Paciente (\%) \\
\hline Osteoblastic & $28(58.3)$ \\
\hline Fibroblastic & $5(10.4)$ \\
\hline Condroblastico & $4(8.3 \%)$ \\
\hline Conventional & $3(6.3)$ \\
\hline Osteogenic & $3(6.3)$ \\
\hline Telangectasico & $I(2 . I)$ \\
\hline Mixed & $4(8.3)$ \\
\hline Grade & Patients (\%) \\
\hline Grade I & $8(16.7)$ \\
\hline Grade 2 & $3(6.3)$ \\
\hline Grade 3 & $37(77.1)$ \\
\hline Enneking Clinical Stage & Patients(\%) \\
\hline Stage IA & $0(0)$ \\
\hline Stage IB & $5(10.4)$ \\
\hline Stage IIA & $6(12.5)$ \\
\hline Stage IIB & $22(45.8)$ \\
\hline Stage III & I5(3|.3) \\
\hline Metastasica & $31(64.5)$ \\
\hline
\end{tabular}

Table 2 Description of treatments used and complications

\begin{tabular}{ll} 
Treatment Modality & Patients (\%) \\
Neoadjuvant Chemotherapy & $25(52.1)$ \\
Adjuvant Chemotherapy & $34(70.8)$ \\
Surgical Amputation & $26(54.2)$ \\
Limb preserving surgery and reconstruction & $21(43.8)$ \\
Complications of Neoadjuvant & Patients (\%) \\
Chemotherapy & $10(20.8)$ \\
Hematological Toxicity. & $2(4.2)$ \\
Infections. & $-*$ \\
Complications of Adjuvant & Patients (\%) \\
Chemotherapy & $9(\mid 8.8)$ \\
Surgical Complications & $8(\mid 6.6)$ \\
Phantom limb syndrome & $5(I 0.4)$ \\
Infectious complications. & $1(2.1)$ \\
Bleeding complicatins & $\mathrm{I}(2.1)$ \\
Prosthesis luxation & \\
Cardiac tamponade & \\
\hline
\end{tabular}
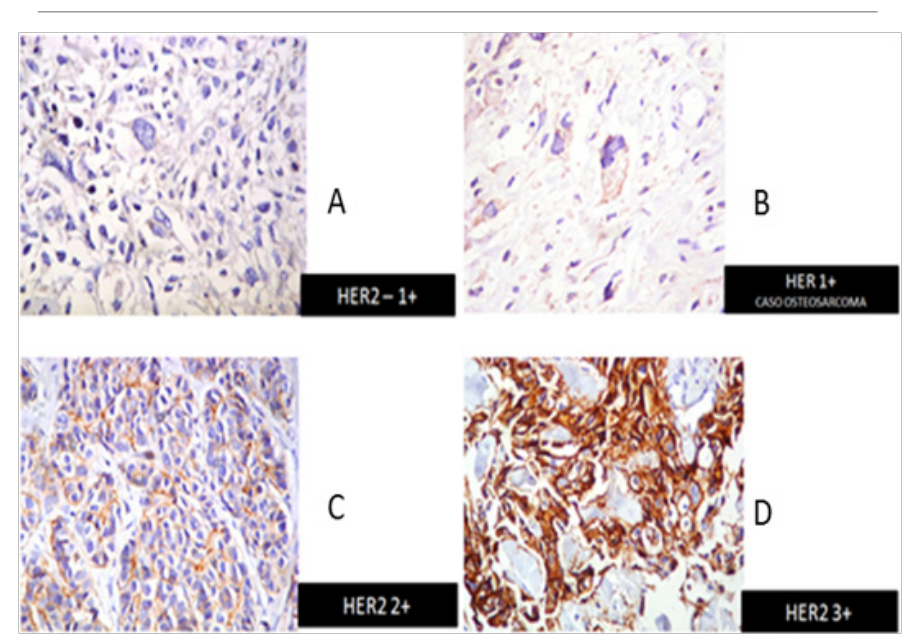

Figure 2 Immunohistochemistry HER2 detection in our patients. A) HER2I+, B) HER I+, C) HER2+, D) HER2 3+.

\section{Discussion}

OS is the most common bone neoplasm, representing around $56 \%$ of all bone malignancies. It also represents around $15 \%$ of all extracranial solid tumors, ${ }^{10}$ and $30-40 \%$ of OS presents with have poor outcomes due to recurrent disease or metastatic disease. ${ }^{17}$ Over the last couple of decades, several new surgical and medical improvements 
have led to improvement in quality of life, overall survival and relapse rate. ${ }^{18,19}$ One of the new potential therapeutic and prognostic target is the overexpression of the Her2 receptor.

Hannayong et al. described a cohort of patient with OS were $57 \%$ of patients overexpressed the Her 2 receptor, with a moderate expression in $12 \%$ of the cohor ${ }^{11}$. Likewise, Onda et al analyzed Her2 overexpression in 26 patient with OS and used several techniques (Western blot, southern blot and in situ hybridization), finding overexpression of the receptor in $42 \%$ of the patients, and a relationship between overexpression and early lung metastasis. ${ }^{10}$

The possible prognostic value of Her2 overexpression was also described by Gorlick et al., ${ }^{12}$ who analyzed 53 cases of OS between 1986 and 1999. In this cohort, Her2 was overexpressed in $42.6 \%$ of cases, and was associated with worse prognosis and less pathologic response. Patients who presented with limited disease and overexpressed Her2 also had a decrease in their 5 years event free survival $(47 \%$ vs $79 \%$ at, $\mathrm{p}=0.05) .{ }^{12}$ These findings were supported by Klemens, who reported Her 2 overexpression in $40-45 \%$ of studied OS, also associated with early lung metastasis and poor chemotherapy response. ${ }^{13}$

However, prognostic value of Her2 is not completely clear, since several researchers have described good outcomes when Her2 is overexpressed. Tomohiro et al. ${ }^{14}$ evaluated 81 non-metastatic (stage II), OS patients, finding 51 cases $(61 \%)$ of Her2 overexpression. Overexpression of the Her2 was associated with improvement in 5 -years event free survival $(72.2 \%$ vs $54 \%, \mathrm{p}=0.03)$, with an overall survival in the cohort of $79.7 \%{ }^{14}$

The present study did not find any case of Her2 overexpression in 48 patients, with $47 / 48$ scoring 0 and only one patient with a score of $1+$ in Her2 IHC score. No interaction was identified between Her2 status and relapse free survival or overall survival. This results goes in line of the findings of Kilpatrick et al, who evaluated 41 patient with OS using H\&E, Her2 IHC and FISH, and could not identify any case of Her2 overexpression and no prognostic value of the marker. ${ }^{16}$

The controversy around Her 2 in OS was also addressed by Anninga et al., ${ }^{15}$ who also described no Her2 overexpression (assessed by IHC, PCR and FISH) in a cohort of OS. These authors also considered that Her2 overexpression assessment in prior studies is unreliable due to use of different monoclonal IHC antibodies and different protocols used during sample processing and staining. They finally concluded that given the lack of Her2 overexpression, therapy with Trastuzumab is unlikely to be effective in this type of tumors. ${ }^{15}$

Due to the multiple conflicting results and different techniques used, the relevance of Her2 overexpression as prognostic or therapeutic marker in OS is doubtful at this point. The first author to describe Her2 overexpression was Onda et al. ${ }^{10}$ in 1996, after analyzing 26 patients by IHC, using the monoclonal antibody CBE 1 , overexpression of the receptor in $42.6 \%{ }^{10}$. Other authors described similar results, with overexpression rate ranging from $40 \%$ to $61 \% .^{12-14}$ However, Onda et al. ${ }^{10}$ did not assess Her2 overexpression with FISH, and other authors used different essays. Additionally, the cutoff and interpretation criteria for IHC is heterogenous (sometimes a positive result defined with different scoring criteria) and often not reported in the some studies. ${ }^{20}$
These heterogeneous results could potentially be explained by different interpretation and preparation of the samples. Thomas et al evaluated patient with OS and Ewing Sarcoma for Her2 overexpression using the rabbit polyclonal antibody A0485, detecting Her2 in 44\% of the cases. However, the IHC staining was seen in the cytoplasm and not in the membrane, which is the known pattern associated with Her2 positivity seen in breast and stomach cancer (and potentially targetable using therapeutic Anti Her2 antibodies. ${ }^{21}$ Supporting this findings, Maitra et al..$^{22}$ assessed 21 cases of pediatric OS using FISH, with no positive case of Her2 overexpression. ${ }^{22}$

The discordance seen in different studies might be related to the type of assay (IHC vs FISH), type of antibody used and processing of sample. This is the case other type of neoplasm and Her2 in breast cancer and PD-L1 in non-small cell lung cancer. ${ }^{23}$ Press et al analyzed 28 Her 2 antibodies in breast cancer ( 7 polyclonal and 21 monoclonal), and described significant variability (6-82\%) between the different antibodies. ${ }^{24}$

Another potentially confounding variable in the case of OS is the use of formalin to fix the sample during the decalcification. All the previously reported studies used formalin, and this could interfere with accurate IHC score. This, in conjunction with variable antibody used and different scoring criteria could potentially explain this conflictive and unclear area.

Possible answers to this issue are the creation and application of standardized process, antibody use and interpretation the Her2 IHC. Also, alternative methods, such as FISH amplification can be used provide further data. However, FISH is not exempt of drawbacks in OS, since this type of tumor frequently have chromosome 17 aneusomy, which can also affect FISH interpretation if the usual control (CEP17) is used. ${ }^{20}$ Also, localization of OS, histological subtype, clinical stage, status of treatment and other clinical variables could contribute to heterogeneous results since most of the studies have different inclusion criteria and sample characteristics.

\section{Conclusion}

The present study showed $100 \%$ absence of Her2 overexpression in a Mexican cohort of patients with OS of the pelvis and extremities, with no relationship with the evaluated clinical outcomes (relapse free survival and overall survival).

These results suggest that Her2 is not overexpressed in patients and does not have any prognostic or therapeutic implication in the management of patient with OS. However, several prior results suggested Her 2 overexpression in around $40-60 \%$ of the patients, with a clear prognostic value. However, given the heterogeneity of clinical variables, different IHC antibodies, interpretation and preparation and other assays, a clear conclusion is not possible at this point.

\section{Acknowledgements}

None.

\section{Conflict of interest}

The main author and other co-authors declare no conflict of interest with the present study. 


\section{References}

1. Society AC. Cancer Facts \& Figures 2016. Atlanta; 2016.

2. Ries LAG, Smith MA, Gurney JG, et al. Cancer Incidence and Survival among Children and Adolescents: United States SEER Program; 19751995.

3. Stiller CA, Bielack SS, Jundt G, et al. Bone tumours in European children and adolescents, 1978-1997. Report from the Automated Childhood Cancer Information System project. Eur J Cancer. 2006;42(13):21242135 .

4. Mirabello L, Troisi RJ, Savage SA. Osteosarcoma incidence and surviva rates from 1973 to 2004: data from the Surveillance, Epidemiology and End Results Program. Cancer. 2009;115(7):1531-1543.

5. http://www.dgis.salud.gob.mx/contenidos/basesdedatos/std defunciones_gobmx.html

6. Dohi O, Hatori M, Suzuki T, et al. Sex steroid receptors expression and hormone-induced cell proliferation in human osteosarcoma. Cancer Sci. 2008;99(3):518-523.

7. Domínguez-Malagón HR, González-Conde E, Cano-Valdez A-M, et al. Expression of hormonal receptors in osteosarcomas of the jaw bones: clinico-pathological analysis of 21 cases. Med Oral Patol Oral Cir Bucal. 2014;19(1):44-48.

8. Rubin I, Yarden Y. The basic biology of HER2. Ann Oncol. 2001;12(Suppl 1):S3-S8.

9. Duda RB, Cundiff D, August CZ, et al. Growth factor receptor and related oncogene determination in mesenchymal tumors. Cancer. 1993;71(11):3526-3530.

10. Onda M, Matsuda S, Higaki S, et al. ErbB-2 expression is correlated with poor prognosis for patients with osteosarcoma. Cancer. 1996;77(1):7178 .

11. Wen YH, Koeppen H, Garcia R, et al. Epidermal growth factor receptor in osteosarcoma: expression and mutational analysis. Hum Pathol. 2007;38(8):1184-1191.

12. Gorlick R, Huvos AG, Heller G, et al. Expression of HER2/erbB-2 correlates with survival in osteosarcoma. J Clin Oncol. 1999;17(9):27812788 .
13. Trieb K, Kotz R. Proteins expressed in osteosarcoma and serum levels as prognostic factors. Int J Biochem Cell Biol. 2001;33(1):11-17.

14. Akatsuka T, Wada T, Kokai Y, et al. ErbB2 expression is correlated with increased survival of patients with osteosarcoma. Cancer. 2002;94(5):1397-1404.

15. Anninga JK, van de Vijver MJ, Cleton-Jansen AM, et al. Overexpression of the HER-2 oncogene does not play a role in high-grade osteosarcomas. Eur J Cancer. 2004;40(7):963-970.

16. Kilpatrick SE, Geisinger KR, King TS, et al. Clinicopathologic analysis of HER-2/neu immunoexpression among various histologic subtypes and grades of osteosarcoma. Mod Pathol. 2001;14(12):1277-1283.

17. Marulanda GA, Henderson ER, Johnson DA, et al. Orthopedic surgery options for the treatment of primary osteosarcoma. Cancer Control. 2008;15(1):13-20.

18. Lamoureux F, Trichet V, Chipoy C, et al. Recent advances in the management of osteosarcoma and forthcoming therapeutic strategies. Expert Rev Anticancer Ther. 2007;7(2):169-181.

19. Sakamoto A, Iwamoto Y. Current status and perspectives regarding the treatment of osteo-sarcoma: chemotherapy. Rev Recent Clin Trials. 2008;3(3):228-231.

20. Judson I. In relation to Anninga et al. Overexpression of the HER-2 oncogene does not play a role in high-grade osteosarcomas. Eur $J$ Cancer. 2004;40(7):924-925.

21. Thomas DG, Giordano TJ, Sanders D, et al. Absence of HER2/neu gene expression in osteosarcoma and skeletal Ewing's sarcoma. Clin Cancer Res. 2002;8(3):788-793.

22. Maitra A, Wanzer D, Weinberg AG, et al. Amplification of the HER-2/ neu oncogene is uncommon in pediatric osteosarcomas. Cancer. 2001;92(3):677-683.

23. Gaule P, Smithy JW, Toki M, et al. A Quantitative Comparison of Antibodies to Programmed Cell Death 1 Ligand 1. JAMA Oncol. $2016 ; 3015$.

24. Press MF, Hung G, Godolphin W, et al. Sensitivity of HER-2/neu antibodies in archival tissue samples: potential source of error in immunohistochemical studies of oncogene expression. Cancer Res. 1994;54(10):2771-2777. 